Pacific Journal of Mathematics

REALIZING CONCORDANT POLYNOMIALS WITH PRIM 


\title{
REALIZING CONCORDANT POLYNOMIALS WITH PRIME KNOTS
}

\author{
Steven A. Bleiler
}

\begin{abstract}
This paper employs the theory of tangles to show that every knot in the 3 -sphere is concordant to a prime knot with the same Alexander polynomial. From this it is shown that all algebraic concordances at the polynomial level are realized by geometric concordances between prime knots.
\end{abstract}

o. Introduction. This paper examines certain geometrical building blocks and uses them to illuminate the relationship between concordances of prime knotes and the Alexander polynomial. Those polynomials in $Z\left[t, t^{-1}\right]$ which can occur as the Alexander polynomial of a knot in the 3-sphere were classified by Seifert [15], see Levine [11]; Fox and Milnor [4] demonstrated that the normalized Alexander polynomials of concordant knots differ by factors of form $f(t) f\left(t^{-1}\right)$, where $f(t)$ is a polynomial in $Z\left[t, t^{-1}\right]$ such that $f(1)=1$. We will call two polynomials in $Z\left[t, t^{-1}\right]$ concordant if they differ in this manner. Using the Conway calculus [3], and a variant of the method recently used by Kirby and Lickorish to prove the primeness of certain knots [9], we establish that every knot is concordant to a prime knot with the same Alexander polynomial. Combining this with some realization theorems of Levine [11], Kondo [10], and Terasaka [16], it will follow that every polynomial concordance can be realized with prime knots.

The author is particularly indebted to W. B. R. Lickorish for indicating the proof of Lemma 1.2, and to the Mathematics Department of the University of California, Santa Barbara for the stimulating atmosphere in which this paper was conceived. The reader is referred to Rolfsen's book [14] for the standard results and definitions of knot theory and should interpret this paper in the PL category.

1. Tangles and the Conway calculus. A tangle is a 3-ball, $T$, containing a finite number of disjoint spanning arcs, called strings,

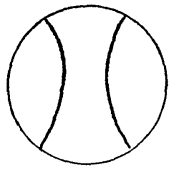

(a)

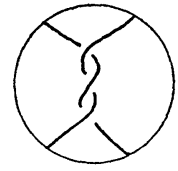

(b)

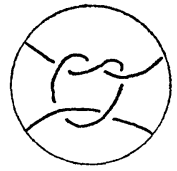

(c)

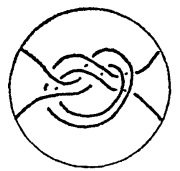

(d)

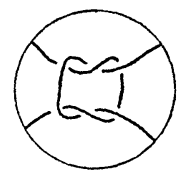

(e)

Figure 1 
which meet $\partial T$ in standard points of an equatorial circle. Some twostring tangles are illustrated in Fig. 1.

Following [9] we define a tangle, $T$, to be prime if

1. Any embedded 2-sphere in $T$ which meets the strings transversely in two points bounds a ball which meets the strings in an unknotted arc.

2. No properly embedded disc separates the strings.

In Fig. 1, (a) and (b) are not prime tangles, (c), (d), and (e) are. Prime tangles are quite common, indeed, we recall from KirbyLickorish [9].

Lemma 1.1. For any knot $K$ in $S^{3}$ there is an embedded 2sphere, meeting $K$ transversely in four points, separating $S^{3}$ into 3-balls $A$ and $B$ such that

(1) $(A, A \cap K)$ is a ball with two standard spanning arcs, a trivial tangle.

(2) $(B, B \cap K)$ is a prime tangle.

In [9], this is first established for prime knots, and then an innermost circle argument establishes that the tangle sum, illustrated in Fig. 2, of prime tangles is also a prime tangle. This gives the lemma.

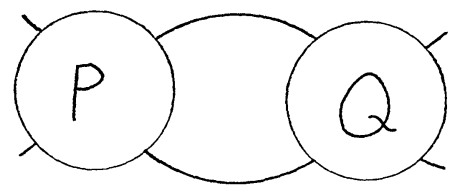

FIGURE 2

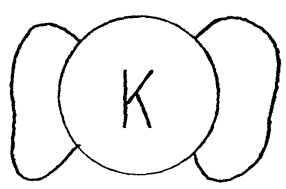

Figure 3

In practice, the lemma asserts that we can consider a knot $K$ as a prime tangle, called " $K$-with-ears", as in Fig. 3. See [9].

The conditions for primeness of a tangle have some nice consequences when combined with some elementary 3-manifold theory. One such is:

LEMMA 1.2. The 2-fold covering space of the 3-ball, $B$, branched over the strings of a prime tangle is an irreducible 3-manifold with an incompressible boundary.

Recall that a surface $F$ in a 3-manifold $M$ is compressible if there exists a disc $D$ in $M$ such that $\partial D=D \cap F$ and $\partial D$ does not bound a disc in $F$. $D$ is called a compressing disc. 
Proof. Let $\tau: M \rightarrow B$ be the branch covering map with decktransformation $h: M \rightarrow M$. If $M$ is not irreducible, it is known [7], [17] that there is a 2 -sphere, $S$ embedded in $M$ such that $S$ does not bound a ball, is transverse to the branch set, Fix $(h)$, and is such that $h(S)=S$ or $h(S) \cap S=\varnothing$.

In the latter case, $F=\tau(S)$ is an embedded 2-sphere in $B$ which misses the strings of our tangle. So $F$ bounds a ball in $B$ which lifts to two balls in $M$, one of which is bounded by $S$, a contradiction.

In the former, we have $\tau \mid S: S \rightarrow F$ a branched covering of surfaces, with finitely many branch points as $S$ is transverse to Fix $(h)$. For an $n$-fold such covering, with branching index $\mu_{i}$ at the $i$ th branch point, the Euler characteristics of $S$ and $F$ are related as in (1.1). See [2] or [12].

$$
\frac{1}{n} \chi(S)=\chi(F)-\sum_{i=1}^{k}\left(1-\frac{1}{\mu_{i}}\right) .
$$

In our case, $n$ and all the $\mu_{\imath}$ equal 2 , and we conclude that $F$ is a 2-sphere meeting the strings of our prime tangle in just two points. As our tangle is prime, $F$ must bound a ball meeting the strings in a single unknotted arc. This has 2-fold branched cover a ball, which must be bounded by $S$, another contradiction, establishing irreducibility.

For the incompressibility of $\partial M$, let $\hat{D}$ be a compressing disc for $\partial M$. Then $\partial \hat{D}$ represents a nontrivial element of $\pi_{1}(\partial M)$ and so appealing to the $Z_{2}$-equivariant form of the Loop Theorem, [7], [17] or more generally [13], we obtain an embedded compressing disc $D$ with $\partial D=\partial \hat{D}$ and either $h(D)=D$ or $h(D) \cap D=\varnothing$.

In the latter case $G=\tau(D)$ is an embedded disc in $B$ with $\partial G$, bounding a disc $E$ in $\partial B$ which misses the branch set. $E$ then lifts to two discs in $\partial M$, one of which is bounded by $\partial D$, contrary to $D$ being a compressing disc.

In the former case, our Euler characteristic formula (1.1) shows that $G$ is a disc with $\tau \mid D: D \rightarrow G$ having one branch point in $G$. So $\partial G$ bounds a disc $E$ in $\partial B$ which also has one branch point. So $G \subset E$ is an embedded 2-sphere which bounds a ball meeting the strings in an unknotted arc. As before, the 2-fold cover is a ball in $M$, the boundary of which is $D$ and a disc in $\partial M$ bounded by $\partial D$, again contrary to $D$ being a compressing disc.

REMARKS. Similar arguments show the incompressibility of the boundary of $M$ where $M$ is now the $n$-fold cyclic cover of the 3 -ball branched over our prime tangle, as the recent work of Meeks and 
Yau [13] gives us a $\boldsymbol{Z}_{n}$-equivariant form of the loop theorem. In addition, should $M$ be the connected sum of nonsimply connected 3-manifolds, [13] also tells us that there is an embedded 2-sphere $S$ in $M$, not homotopically trivial, such that $h(S) \cap S=\varnothing$ for all nontrivial $h$ in the group of deck transformations of $M$ or $h(S)=S$ for all such $h$. Now, modulo the existance of a fake 3-ball, an argument similar to the case where $n=2$ establishes that the $n$-fold cyclic covering space of the 3-ball branched over the strings of a prime tangle is an irreducible 3-manifold with an incompressible boundary.

Given prime tangles $P$ and $Q$, we define their join, $P \vee Q$, as the knot or link obtained by identifying the boundaries of $P$ and $Q$ in such a way that $P$ and $Q$ are connected by "bands". See Fig. 4.

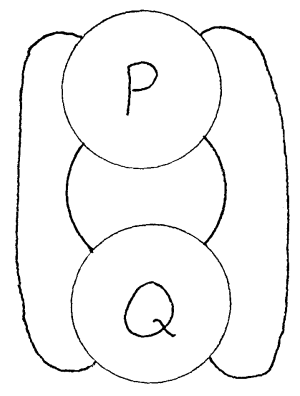

FiguRE 4

If $M$ is now the 2-fold cover of $S^{3}$ branched over the join of two prime tangles, we see that the 2 -sphere which separates $S^{3}$ into our prime tangles lifts to an incompressible torus $T$ in $M$. Any embedded 2-sphere in $M$ which has been isotoped to have minimal transverse intersection with $T$ has no "innermost" component, and so we see that $M$ is irreducible. This shows, using [7],

Proposition 1.3. The join of two prime tangles is a prime knot or a prime link.

Until now we have been unconcerned with orientations, but these play an important role in computing the Alexander polynomial.

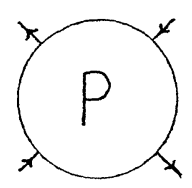

FiguRE 5
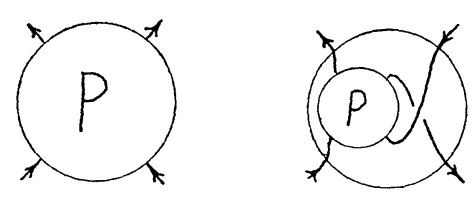

Figure 6 
We wish our tangles to carry the string orientations as shown in Fig. 5. Fig. 6 shows that we can assume that the prime tangles given by Lemma 1.1 carry this string orientation. Notice also that the join of prime tangles with these orientations is now an oriented knot or link.

For an oriented link $L$ in $S^{3}$, Conway [3] defines an integer polynomial, $\nabla_{L}(z)$, the Conway potential function. When the chance of confusion is slight we will write $\nabla(L)$ for $\nabla_{L}(z)$. For knots, the potential function is related to the usual Alexander polynomial, $\Delta_{K}(t)$, normalized so that $\Delta_{K}(1)=1$, via $\nabla_{K}\left(t^{1 / 2}-t^{-1 / 2}\right)=\Delta_{K}(t)$.

The Conway potential function can be computed recursively, beginning with $\nabla$ (unknot) $=1, \nabla$ (unlink of two unknotted components $)=0$, and using Conway's first identity:

$$
\nabla_{R}(z)=\nabla_{L}(z)+z V_{S}(z)
$$

where $R, L$, and $S$ are the links differing at a single crossing as indicated in Fig. 7. See [3] or [5].

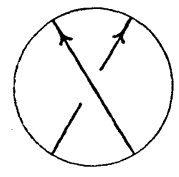

$R$

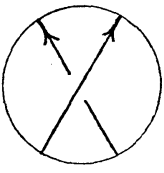

$L$

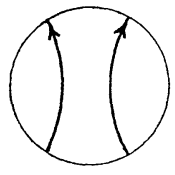

$S$

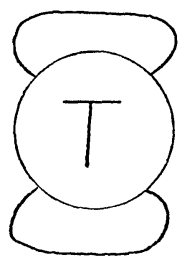

(a)

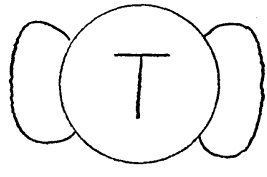

(b)

FiguRe 7

Figure 8

There is also a formula for handling oriented two-string tangles, the determinant fraction of a tangle sum. Let $\nabla_{N}(T), \nabla_{D}(T)$ be the Conway potential function of the link obtained by completing the tangle $T$ as in Fig. 8a, Fig. 8b respectively. For tangles oriented as in Fig. 9 (note the difference with Fig. 5), the determinant fraction, given in formulas 2.2 and 2.3 is a pair of formulas for the potential function of the tangle sum given in terms of the potential functions of the tangles themselves. Again see [3] or [5].

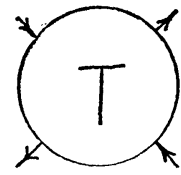

FIGURE 9 


$$
\begin{gathered}
\nabla_{N}(P+Q)=\nabla_{N}(P) \nabla_{D}(Q)+\nabla_{D}(P) \nabla_{N}(Q) \\
\nabla_{D}(P+Q)=\nabla_{D}(P) \cdot \nabla_{D}(Q)
\end{gathered}
$$

We wish to examine the case of $P \vee Q$, the join of two prime tangles oriented as in Fig. 5. Fig. 10 shows that $P \vee Q$ is just the
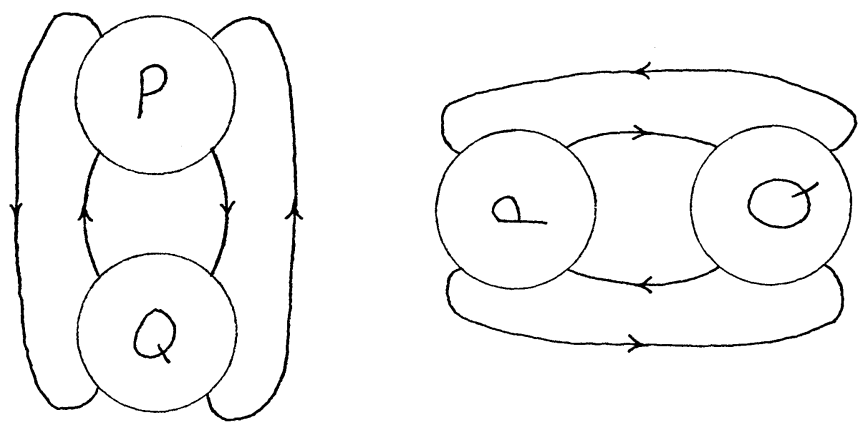

Figure 10

"numerator" of the tangle sum of the prime tangles $i P$ and $i Q$, obtained by rotating $P$ and $Q$, respectively, through 90 degrees. Note that $i P$ and $i Q$ carry the orientations of Fig. 9 and thus those of [3] and [5], so we can apply (2.2) to obtain

$$
\nabla(P \vee Q)=\nabla_{N}(i P) \nabla_{D}(i Q)+\nabla_{D}(i P) \nabla_{N}(i Q)
$$

or more usefully

$$
\nabla(P \vee Q)=\nabla_{D}(P) \cdot \nabla_{N}(Q)+\nabla_{N}(P) \cdot \nabla_{D}(Q)
$$

2. Some results. A particularly useful tangle is the $K-T$ grabber, pictured in Fig. 11a, and here occasionally denoted by $K T$.

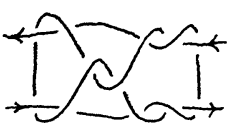

(a)

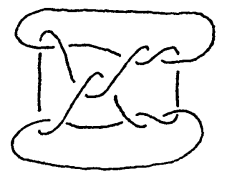

(b)

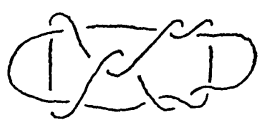

(c)

FIGURE 11

The name is suggested by its use and the fact that $K T$ has as its "numerator" a twelve crossing projection of the eleven crossing Kinoshita-Terasaka knot, which has Alexander polynomial 1, Fig. $11 \mathrm{~b}$. Further, $K T$ has as its "denominator" the unlink of two trivial components, Fig. 11c, and so we see that $\nabla_{N}(K T)=1$ and $\nabla_{D}(K T)=0$.

LEMmA 2.1. The K-T grabber is a prime tangle. 
Proof. Condition 1 is immediate as each spanning arc is unknotted. If condition 2 failed, the Kinoshita-Terasaka knot would be a 2-bridge (rational) knot. We assert that such a knot cannot enjoy a trivial Alexander polynomial. For the 2 -fold branched cover $M$ of a 2-bridge knot $K$ is a Lens Space, implying that the determinant, $\left|H_{1}(M)\right|=\left|\Delta_{K}(-1)\right|$, is greater than 1 .

Notice also that $K-T$ grabber carries the string orientations of Fig. 5. We are now ready to prove

THEOREM 2.2. Any knot $K$ in $S^{3}$ is (ribbon) concordant to a prime knot with the same Alexander polynomial.

Proof. Apply Lemma 1.1 to $K$, to get a prime tangle, called " $K$-with-ears", carrying the string orientations of Fig. 5. Now join the $K-T$ grabber to $K$ and call the resulting knot $L$. See Fig. 12.

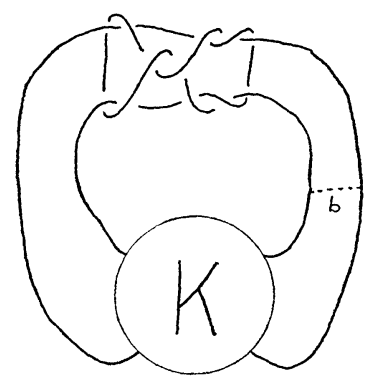

Figure 12

Notice that a ribbon move at $b$ shows that $L$ is (ribbon) concordant to $K$. The primeness of $L$ follows from Lemma 1.3 and applying formula (2.5) we see that

$$
\begin{aligned}
\nabla_{L}(z) & =\nabla_{N}(K T) \nabla_{D}(K)+\nabla_{D}(K T) \nabla_{N}(K) \\
& =1 \cdot \nabla_{K}(z)+0 \cdot \nabla_{N}(K)=\nabla_{K}(z) .
\end{aligned}
$$

And so $\Delta_{K}(t)=\Delta_{L}(t)$.

REMARK. The $K-T$ grabber is not the only prime tangle that could be used in the proof of the above theorem. There is, in fact, an infinitude of such tangles. For more on this, see [2].

Our realization results now follow easily.

CoROLLARY 2.3. Given $f(t)$ in $Z\left[t, t^{-1}\right]$ with $f(1)=1$, there is a prime ribbon knot $R$ with $\Delta_{R}(t)=f(t) f\left(t^{-1}\right)$. 
Proof. Apply Terasaka's method [14] to obtain a ribbon knot $K$ with $\Delta_{K}(t)=f(t) f\left(t^{-1}\right)$. Apply Theorem 2.2 to $K$ to obtain $R$.

CoROLLARY 2.4. Given polynomials $g(t), f(t)$ in $Z\left[t, t^{-1}\right]$ satisfying $g(t)=g\left(t^{-1}\right)$ and $g(1)=f(1)=1$, there exist prime concordant knots $K, L$ with $\Delta_{K}(t)=g(t)$ and $\Delta_{L}(t)=g(t) f(t) f\left(t^{-1}\right)$.

Proof. Use the surgery method of Levine [11], clearly outlined in [14], to obtain a knot, $\hat{K}$, realizing $g(t)$. Alternately, one can use the band method seen in Kondo [10] to obtain $\hat{K}$. The knot given by the latter method has unknotting number equal to 1 , and is thus very close to being prime. If not, however, just apply Theorem 2.2 to get the knot $K$. Next obtain a slice knot with Alexander polynomial $f(t) f\left(t^{-1}\right)$ and take the connected sum of this knot and $K$. The resulting knot has $g(t) f(t) f\left(t^{-1}\right)$ for its Alexander polynomial and is concordant to $K$ as well. Applying Theorem 2.2 to this knot gives $L$.

Combining 2.3 and 2.4 we have shown, as advertised in the introduction:

THEOREM 2.5. Every polynomial concordance is realized by prime knots.

\section{REFERENCES}

1. J. W. Alexander, Topological invariants of knots and links, Trans. Amer. Math. Soc., 30 (1928), 275-306.

2. S. A. Bleiler, $\mathrm{Ph}$. D. Thesis, University of Oregon.

3. J. H. Conway, An Enumeration of Knots and Links, Computational Problems in Abstract Algebra, Pergamon Press, Oxford and New York, (1969), 329-358.

4. R. H. Fox and J. W. Milnor, Singularities of 2-spheres in 4-space and cobordisms of knots, Osaka J. Math., 3 (1966), 257-267.

5. C. A. Giller, A family of links and the Conway calculus, to appear.

6. C. McA. Gordon, Ribbon concordances of knots in the 3-sphere, to appear.

7. P. Kim and J. Tollefson, Splitting the PL involutions on non-prime 3-manifolds, Michigan Math. J., 27 (1980), 259-274.

8. S. Kinoshita and H. Terasaka, On unions of knots, Osaka Math. J., 9 (1959), 131-153.

9. R. C. Kirby and W. B. R. Lickorish, Prime knots and concordance, Math. Proc. Camb. Phil. Soc., 86 (1979), 437-441.

10. H. Kondo, Knots of unknotting number 1 and their Alexander polynomials, Osaka J. Math., 16 (1979), 551-559.

11. J. Levine, A characterization of knot polynomials, Topology, 4 (1965), 135-141.

12. W. B. R. Lickorish, 3-manifolds - a course at UCSB, winter 1980, notes by S. A. Bleiler.

13. W. H. Meeks and S. T. Yau, Topology of 3-dimensional manifolds and the embedding problems in minimal surface theory, Ann. of Math., 112 (1980), 441-484.

14. D. Rolfsen, Knots and Links, Publish or Perish, 7, Berkeley, CA, 1976. 
15. H. Seifert, Über das Geschlecht von Knoten, Math. Ann., 110 (1934), 571-592.

16. H. Terasaka, On null-equivalent knots, Osaka Math. J., 11 (1959), 95-113.

17. F. Waldhausen, Über involutionen der 3-sphäre, Topology, 8 (1969), 81-91.

Received August 8, 1980.

University of Oregon

Eugene, OR 97403 



\section{PACIFIC JOURNAL OF MATHEMATICS}

\section{EDITORS}

DONALD BABBITT (Managing Editor)

University of California

Los Angeles, CA 90024

Hugo RossI

University of Utah

Salt Lake City, UT 84112

C. C. Moore and Arthur Agus

University of California

Berkeley, CA 94720
J. DugundJI

Department of Mathematics

University of Southern California

Los Angeles, CA 90007

R. FinN and J. MiLgRAM

Stanford University

Stanford, CA 94305

\section{ASSOCIATE EDITORS}
R. ARENS
E. F. BECKENBACH
B. H. NeumanN
F. WOLF
K. YoSHIDA

\section{SUPPORTING INSTITUTIONS}

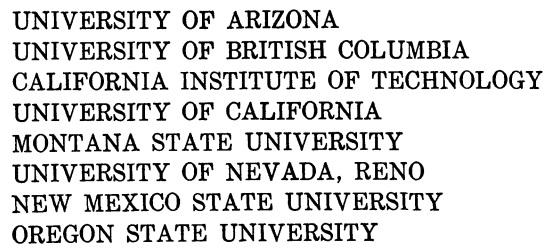

UNIVERSITY OF ARIZONA

UNIVERSITY OF BRITISH COLUMBIA CALIFORNIA INSTITUTE OF TECHNOLOGY UNIVERSITY OF CALIFORNIA MONTANA STATE UNIVERSITY

UNIVERSITY OF NEVADA, RENO NEW MEXICO STATE UNIVERSITY OREGON STATE UNIVERSITY

\author{
UNIVERSITY OF OREGON \\ UNIVERSITY OF SOUTHERN CALIFORNIA \\ STANFORD UNIVERSITY \\ UNIVERSITY OF AAWAII \\ UNIVERSITY OF TOKYO \\ UNIVERSITY OF UTAH \\ WASHINGTON STATE UNIVERSITY \\ UNIVERSITY OF WASHINGTON
}

The Supporting Institutions listed above contribute to the cost of publication of this Journal, but they are not owners or publishers and have no responsibility for its content or policies,

Mathematical parers intended for publication in the Pacific Journal of Mathematics should be in typed form or offset-reproduced, (not dittoed), double spaced with large margins. Please do not use built up fractions in the text of the manuscript. However, you may use them in the displayed equations. Underline Greek letters in red, German in green, and script in blue. The first paragraph or two must be capable of being used separately as a synopsis of the entire paper. Please propose a heading for the odd unmbered pages of less than 35 characters. Manuscripts, in triplicate, may be sent to any one of the editors. Please classify according to the scheme of Math. Reviews, Index to Vol. 39. Supply name and address of author to whom proofs should be sent. All other communications should be addressed to the managing editor, or Elaine Barth, University of California, Los Angeles, California, 90024 .

50 reprints to each author are provided free for each article, only if page charges have been substantially paid. Additional copies may be obtained at cost in multiples of 50 .

The Pacific Journal of Mathematics is issued monthly as of January 1966, Regular subscription rate: $\$ 114.00$ a year (6 Vol., 12 issues). Special rate: $\$ 57.00$ a year to individual members of supporting institution.

Subscriptions, orders for numbers issued in the last three calendar years, and changes of address shoud be sent to Pacific Journal of Mathematics, P.O. Box 969, Carmel Valley, CA 93924, U.S.A. Old back numbers obtainable from Kraus Periodicals Co., Route 100, Millwood, NY 10546.

\section{PUBLISHED BY PACIFIC JOURNAL OF MATHEMATICS, A NON-PROFIT CORPORATION}

Printed at Kokusai Bunken Insatsusha (International Academic Printing Co., Ltd.). 8-8, 3-chome, Takadanobaba, Shinjuku-ku, Tokyo 160, Japan.

Copyright (C) 1982 by Pacific Journal of Mathematics Manufactured and first issued in Japan 


\section{Pacific Journal of Mathematics}

Vol. 100, No. $2 \quad$ October, 1982

Kenneth F. Andersen, On the transformation of Fourier coefficients of

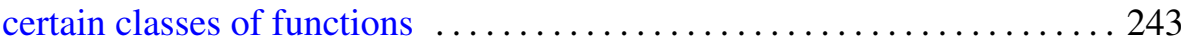

Steven Albert Bleiler, Realizing concordant polynomials with prime knots

Reinhard Bürger, Functions of translation type and solid Banach spaces of functions

Ulrich Daepp, The saturation of $k$-analytic rings and topological equivalence of associated analytic set germs .................. 271

Persi W. Diaconis and David Amiel Freedman, On the maximum difference between the empirical and expected histograms for sums . . . 287

David Amiel Freedman, On the maximum of scaled multinomial

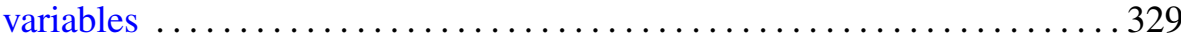

Persi W. Diaconis and David Amiel Freedman, On the difference between the empirical histogram and the normal curve, for sums. II ......... 359

Persi W. Diaconis and David Amiel Freedman, On the mode of an

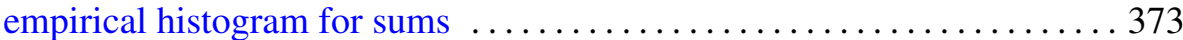

Jutta Hausen, Supplemented modules over Dedekind domains 387

Elyahu Katz, A moduli representation for the classification of twisted tensor

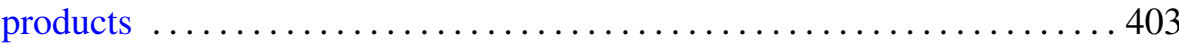

H. C. Madhekar and N. K. Thakare, Biorthogonal polynomials suggested by the Jacobi polynomials

Ted R. Pettis, Collections of covers of metric spaces 425

Ryōtarō Satō, Maximal functions for a semiflow in an infinite measure space

Michael Jay Stob, Invariance of properties under automorphisms of the lattice of recursively enumerable sets 